\title{
eJRIEPS
}

Ejournal de la recherche sur l'intervention en éducation physique et sport

$10 \mid 2006$

Varia

\section{Du conflit à la prise d'initiative : le sujet a l'épreuve du combat en judo}

\section{Marc Galan, André Terrisse et Patricia Vigne}

\section{OpenEdition}

\section{Journals}

Édition électronique

URL : https://journals.openedition.org/ejrieps/7279

DOI : 10.4000 /ejrieps.7279

ISSN : 2105-0821

Éditeur

ELLIADD

Édition imprimée

Pagination : 34-53

\section{Référence électronique}

Marc Galan, André Terrisse et Patricia Vigne, « Du conflit à la prise d'initiative : le sujet a l'épreuve du combat en judo », eJRIEPS [En ligne], 10 | 2006, mis en ligne le 01 juillet 2006, consulté le 29 octobre 2021. URL : http://journals.openedition.org/ejrieps/7279; DOI : https://doi.org/10.4000/ejrieps.7279

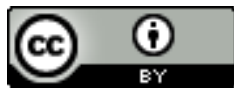

La revue eJRIEPS est mise à disposition selon les termes de la Creative Commons Attribution 4.0 International License. 


\title{
Du conflit à la prise d'initiative : le sujet a l'épreuve du combat en judo.
}

\author{
Marc Galan*, André Terrisse*, \& Patricia Vigne** \\ *Université Paul Sabatier, Toulouse III, EA 3692, LEMME, AP3E \\ **Université Toulouse II Le Mirail, EA 1697, PCS.
}

\section{Résumé}

Cette recherche en didactique des sports de combat concerne le processus d'enseignement/apprentissage du judo dans le contexte d'une classe de Lycée professionnel, pendant un cycle d'Education Physique et Sportive. Elle a pour objet l'analyse des rapports pouvant exister entre la mise en scène par un enseignant d'une option didactique, définie en terme d'initiative, les conduites des élèves lors de l'épreuve du combat et l'évaluation qui peut en être faite.

La méthode de l'ingénierie didactique, (Artigue, 1988) associée à des outils d'investigations cliniques, constitue le cadre à partir duquel nous tentons de décrire, de comprendre et de proposer une signification relative à ce que fait le sujet "apprenant » autrement qu'en terme d'écarts par rapport à ce qui était attendu par l'enseignant.

La pratique sportive peut conduire le sujet pratiquant vers des situations qui peuvent s'avérer difficiles pour lui.

Si le sport de haut niveau constitue l'archétype de la difficulté sportive, l'éducation physique et sportive (EPS) peut conduire certains élèves vers des situations qu'ils peuvent percevoir comme difficiles, voire « trop difficiles ». La difficulté objective de la tâche n'est plus la seule référence. Intervient également la représentation par l'élève de l'écart existant entre son " déjà là » (ce qu'il sait déjà faire) et les caractéristiques de la situation qui lui pose problème.

Dès lors, la notion d'épreuve (associée à la compétition, celle de la mise à l'épreuve de son savoir par le sujet) occupe une place essentielle dans ces deux domaines d'activité que sont le sport et l'EPS.

Dans le secteur du sport de haut niveau, c'est la performance de l'athlète dans l'activité sportive choisie qui est significative de sa compétence et... de celle de l'équipe technique qui l'accompagne. L'épreuve est souvent confondue avec le résultat: il s'agit d'être le premier. Quelle est alors la place du sujet et de son savoir dans une telle dynamique ? Les 
exemples de ruptures entre les attentes des sportifs de haut niveau et les conditions de leur prise en charge sont aujourd'hui médiatisés et semblent récurrents. Suffiront-ils à bousculer les routines d'entraînement et à considérer les athlètes comme des sujets, c'està-dire capables d'initiative?

En éducation physique et sportive, les enseignants doivent favoriser l'appropriation par l'élève de compétences lui permettant, en fin de cycle, de faire face à des situations jugées signifiantes au regard des problèmes qui se posent à lui et de la "culture » de l'activité proposée. La notion d'épreuve est au centre du processus d'enseignement/apprentissage en EPS, dans la mesure où elle est une mise à l'épreuve des ressources de l'élève, d'autant plus significative que l'activité physique sportive ou artistique n'est généralement pas choisie par lui. Ce dernier l'investit, non seulement en fonction des caractéristiques objectives de l'activité ou des tâches proposées par l'enseignant, mais aussi au gré de ses « conceptions » (Giordan, 1989) et des ressources qui lui sont propres. Par conséquent, les situations proposées par l'enseignant à l'élève peuvent a priori être significatives d'appétence ou de rejet vis-à-vis de l'activité. La situation didactique peut alors devenir le lieu d'expression de multiples obstacles, voire de conflits, qui émergent chez le sujet (élève et/ou enseignant) et induisent des écarts entre : les savoirs mis en scène par l'enseignant et les représentations de l'élève, la difficulté de la tâche et le sentiment perçu par l'élève de pouvoir y faire face, le savoir enseigné et le savoir mobilisé par l'élève au moment de l'épreuve, ses productions et celles des autres...

Existe-t-il, dans ce contexte scolaire d'enseignement/apprentissage, un lien causal entre l'intention de l'enseignant, la dynamique de l'élève concernant la gestion d'une situation qui peut s'avérer plus ou moins difficile, et l'appropriation de compétences ? En quoi la part que prend l'élève au moment de l'épreuve lui permet d'accéder au savoir combattre ? Est ce que l'enseignant peut et doit la prendre en compte ou l'ignorer dans son projet d'enseignement?

\section{Problématique de recherche}

Dans ces deux situations (compétition et enseignement), il est notoire de constater des écarts entre les effets attendus par l'entraîneur ou par l'enseignant et les réalisations effectives des athlètes ou des élèves au moment de l'épreuve. La description et l'exploration de ces écarts, dans le contexte fourni par l'éducation physique et sportive, est l'enjeu de plusieurs recherches en sport de combat (Sauvegrain, 2001, Heuzer, 2005). 
Notre recherche s'inscrit dans la continuité de ces travaux et se centre sur le «travail de l'élève » (Brousseau, 1986) confronté à l'épreuve du combat en judo, pour apprécier l'effet de l'enseignement. Elle s'appuie sur la définition, puis la négociation avec l'enseignant qui la mettra en scène, d'une option didactique référée à la notion d'initiative. Cette définition justifiée du savoir, en référence avec le concept de transposition didactique (Verret, 1975), introduit une rupture par rapport aux options didactiques usuelles, évoquées ci-après. Elle restitue à l'élève sa place, et sa fonction dans l'appropriation du « savoir combattre en judo $"$.

L'analyse des effets sur des élèves de cette option d'enseignement supposée créer les conditions d'un accès possible à une dynamique de sujet " combattant », nous permet de qualifier, autrement qu'en terme d'écart, le savoir mobilisé par l'élève au moment de l'épreuve. Cette conception de la recherche n'est pas sans incidence sur le recueil de données issues du combat. En effet, l'analyse du combat par l'enseignant doit aller au delà des seuls repères biomécaniques, techniques et décisionnels traditionnels, et s'attacher à révéler la dynamique du sujet, au travers de stratégies, de comportements, d'attitudes qui lui permettent de faire face, ou non, à la difficulté, à l'adversité, au contexte d'opposition... Dans la mesure où « seul le sujet peut s'interroger sur ce qu'il est luimême » (Alberti, Sauret, 1997), cette exploration implique, pour les différents acteurs (enseignant, entraîneur, élève, athlète, chercheur), la prise en compte d'un temps de réflexion et de construction « après coup ».

\section{Le judo : du savoir de référence à la logique motrice (Parlebas, 1993)}

\section{1. Le savoir en judo}

La définition, la sélection et l'évaluation du savoir constituent des étapes essentielles de la transposition didactique du judo en milieu scolaire. Elles portent le sceau des conceptions de l'enseignant concernant le judo et induit de manière corollaire un mode particulier de prise en compte du travail de(s) (l') élève(s). Cette phase revêt une acuité d'autant plus sensible qu'il n'existe de savoir dit « savant » ni en EPS ni en judo. II s'agit d'un savoir pratique, c'est-à-dire " essentiellement un savoir qui s'exécute, qui ne se dit ni ne s'écrit » (Van der Maren, 1996). La définition de la forme de pratique qui fait référence s'impose étant donné la multiplicité des usages sociaux du judo (éducation, compétition, self défense, activité physique de détente ou d'entretien); le savoir de référence est ici la « référence experte » (Joshua, 1996) issue des acteurs de la «pratique sociale de référence » (Martinand, 1989). 
Nous choisissons donc de nous intéresser à la forme sportive du judo. Cette visée, un temps (souvent ?), décriée par les «traditionalistes », ne nous semble pas en rupture avec l'enseignement originel du judo. En effet, "l'art du détail » (Brousse, 1994) qui a permis au judo de s'implanter en France et de se distinguer socialement et quantitativement de la trajectoire de la lutte fait toujours loi. La conception « technique » de cette activité semble participer, pour une grande part, à son identité. Elle se retrouve tant dans les ouvrages spécialisés (Badreau, 1994), que dans les pratiques d'enseignement en milieu fédéral (Loizon, 2001).

Lorsqu'il intègre le milieu scolaire, le judo reste assujetti à cette conception " technique » de son enseignement; de fait, il y est peu enseigné. La spécificité du contexte d'enseignement scolaire (volume d'enseignement peu important, formation restreinte des enseignants, représentation technique de l'activité) de cette activité semble, alors, cautionner des simplifications suggérées dans les propositions didactiques concernant l'enseignement du judo en EPS : « l'accès à une forme globale de combat réel de lutte ou de judo, avec des élèves qui ne les pratiquent qu'en EPS » est impossible (Barbot, 1999). Aussi, les propositions didactiques actuelles semblent devoir s'orienter vers une alternative entre deux types d'option didactique, que nous allons développer:

- une option duelle : Barbot milite pour « une EPS culturalisée et contextualisée » (Barbot, 1996). Il ne retient, comme objet d'enseignement des « sports de combat de préhension », que la construction de la garde et l'utilisation de la force de l'adversaire dans un combat. Pour mettre ces savoirs en évidence, il propose une épreuve, «la lutte au cercle », excluant de son règlement toute possibilité de projection... et ses répercussions symboliques et techniques.

- une option centrée sur les exercices traditionnels ; à l'opposé, Calmet (2000) refuse la mise en avant prépondérante des notions de victoire, de domination ou d'imposition d'un état de force. Pour rendre compte des buts d'apprentissage et de résultats en coopération et en opposition, il choisit deux situations d'évaluation se référant aux « conceptions historiques du judo »: les kata témoins d'un «savoir faire avec » et les randori caractérisant le " savoir faire contre ».

Nous retrouvons cette dernière conception combinée de l'épreuve, permettant l'appréciation juxtaposée des acquisitions «techniques » et de la combativité, dans les modalités récentes d'évaluation de la pratique du judo en Lycée (M.E.N., 2001)... Suffitelle à rendre compte de la spécificité de la tâche du judoka, de sa compétence à mobiliser des acquisitions techniques au moment du combat ? N'induit-elle pas, du fait de sa 
conception dichotomique (l'avec puis le contre), l'adoption par l'élève de registres de savoirs différents?

2. 2. La spécificité du judo : une dialectique entre le sujet et l'objet de savoir. L'enseignement de savoirs d'ordre technique, stratégique ou éthique est essentiel pour les professeurs de judo (Loizon, Margnes, Terrisse, 2004). Ces savoirs identifiés au travers de la mise en œuvre des variables didactiques par ces professionnels de l'enseignement du judo constituent les assises de l'activité. Pourtant, la juxtaposition de ces savoirs n'est pas le judo...

En effet, au moment du combat, le judoka doit actualiser ses ressources dans un contexte inédit, jamais totalement prévisible. Le combat est caractérisé par une forte incertitude, inhérente à l'intention adverse et par une temporalité supposant, dans l'alternative « gagner sinon perdre », des prises de décisions dans l'urgence. « Si le judoka réalise donc bien un travail sur Soi, celui-ci dépend en permanence d'un travail sur l'Autre, l'adversaire, dans le cadre d'une opposition véritable dont l'issue, incertaine, est constamment remise en cause »(Bui-Xuan, 1993). L'autre est porteur de significations d'action : «agir, c'est réagir, souvent même pré-agir » (Parlebas, 1981). Les choix effectués dans une situation de crise temporelle ont une charge émotionnelle importante ; ils peuvent être à l'origine de la victoire ou bien être exploités par l'autre et signifier sa défaite. Durand, Geoffroi et Jacquemond identifient deux stratégies qui permettraient au sportif expert «d'échapper aux impératifs d'une réactivité aux sollicitations de l'environnement : 1) en repérant des régularités dans les modifications de l'environnement à partir desquelles il pré-sélectionne et pré-programme ses réponses ; 2) en construisant un répertoire de schèmes permettant de lire la situation actuelle et d'anticiper à court terme les évènements sur la base d'une prise d'information non sur les actions de l'adversaire mais sur les siennes »(Durand, Geoffroi et Jacquemond, 1993). Dans cette dynamique, ce n'est plus le contexte qui imposerait une réaction au combattant, c'est le combattant qui est « avant » : il anticipe, il (est) à l'initiative.

Dès lors, il semble nécessaire que les situations d'entraînement/d'enseignement, les informations, les évaluations prennent en compte les ressources singulières et l'espace de signification du combattant. Pour Rochex, cette question du sens pour et par le sujet exprime « le rapport qui existe entre ce pourquoi on agit et ce que l'on fait réellement (...) le sens est donc l'interface entre l'ici et maintenant de l'activité, son versant objectif, qui se donne à voir en termes d'actions et d'opérations, évaluables du point de vue de leur efficacité et de leur efficience, et son versant subjectif qui, lui, se dérobe non seulement au 
regard de l'observateur, mais bien souvent à la conscience même du sujet » (Rochex, 1995).

Parlebas fait appel au concept de « logique motrice » pour rendre compte du « rapport que le pratiquant entretient avec le milieu, avec les engins, avec autrui : ces formes de rapport lui sont certes imposées par le code, mais il les vit au travers de sa propre personnalité, de ses expériences antérieures, de ses émotions singulières. Ainsi la logique interne est-elle au confluent des contraintes objectives du système et des ressources subjectives de l'acteur. Elle n'impose pas les comportements des joueurs, mais elle les probabilise.» (Parlebas, 93).

Au moment de l'épreuve, la prise en compte de la singularité du sujet s'avère incontournable, et doit être appréciée au-delà des seules différences concernant la production technique et/ou la modélisation stratégique. II convient donc de se détacher du produit, l'action qui fait gagner, pour s'intéresser à son émergence, en situation, par un sujet.

\section{L'initiative: interface entre les ressources internes du sujet et les logiques internes du judo}

3. 1. Justification de la notion d'initiative dans cette recherche en judo

L'analyse des prestations de judokas de haut niveau nous a conduit à identifier le thème de l'initiative (sa prise, sa reprise et sa conservation) comme critère significatif de performance. Les systèmes d'attaques sont différenciés, l'intention est la même : faire avant, être devant, être le premier! Ainsi, la gestion de cette situation difficile qui caractérise le combat, exige d'abord cette croyance en sa capacité à faire face, à trouver et mettre en œuvre sa solution...

J.L. Rougé, premier champion du monde français et actuel président de la FFJDA, témoigne de la nécessaire construction de cette attitude pour gagner : " personne n'allait jusqu'au bout face à eux (Les Japonais), c'était conscient ou inconscient, c'était pas forcément tout à fait calculé, car vu l'aura des japonais, vu l'histoire... A Vienne, nous avons tous décidé, le monde entier, de dire stop. C'était pas un mot d'ordre mais tout simplement une prise de conscience ... et nous avons remporté des titres de champions du monde » (Rougé, 1997).

La capacité à s'engager de manière déterminée « avec/contre » l'autre semble constituer un préalable au combat et entretien un lien d'inter-structuration avec le « savoir combattre » (Terrisse, 1996). Par ailleurs, l'engagement d'adolescents dans des situations sportives pouvant s'avérer conflictuelles, et la mise en jeu, sur un mode actif, de stratégies 
et variables psychologiques (estime de soi, locus of control, coping, compétence sociale) sont aussi corrélés et participeraient du processus de personnalisation (Vigne, 1999). C'est dans ce sens qu'il faut comprendre le commentaire concernant la pratique des sports de combat au collège : elle "doit permettre à l'élève d'exprimer sa volonté de vaincre un adversaire dans le respect de l'éthique d'affrontement en contrôlant ses actions et ses émotions » (M.E.N., 1997).

\section{2. Définitions}

L'initiative est, certes, "l'action de celui qui propose ou qui fait le premier quelque chose. ", mais c'est d'abord, la "qualité de celui qui est porté à agir, à entreprendre spontanément. Prendre l'initiative, c'est devancer dans une affaire" (Larousse, 2002). Cette notion n'est pas nouvelle dans le domaine du judo puisque pour J. Kano, le fondateur du judo, le principe lié à la prise d'initiative constitue l'un des cinq principes qui «peut s'appliquer tant au judo qu'à la vie quotidienne». Pour l'illustrer, il évoque la stratégie mise en œuvre par les joueurs d'échecs ou de go « qui consiste à déplacer une pièce qui obligera l'autre joueur à agir d'une façon donnée » (Kano, 1956).

L'initiative peut donc caractériser l'intention du combattant. A ce titre, elle pourrait constituer un filtre favorisant l'accès à une description, une compréhension, une explication voire une évaluation de ses stratégies et comportements développés en situation de combat. L'initiative qualifie les réponses mises en œuvre par le sujet pour faire face à la complexité inhérente au combat. Ainsi, il n'y a plus de bonne solution a priori, mais une solution pertinente parce qu'elle aura permis au combattant d'être « devant » au terme de l'action et/ou du combat. La solution est toujours à édifier in situ dans l'interaction avec le combattant adverse. Une solution ayant réussi lors de combats précédents ne peut être importée directement (comme plaquée) dans un autre combat au risque d'échouer.

Les analyses des combats communément effectuées en terme d'efficacité, de diversité technique, de rapport de domination ou même en terme de prise de décisions, se focalisent souvent sur ce qui est extérieur à l'athlète (le savoir idéalisé, l'adversaire, le score, un modèle...). Nous pensons que prendre en compte l'initiative peut conduire à un changement de point de vue, où le mode de fonctionnement spécifique du sujet pourrait constituer le point d'ancrage de toute tentative explicative, donc d'enseignement et d'entraînement.

Tout comme la performance qui la met en valeur, elle se situe "sur le fil de l'interface entre, d'un coté, le vécu subjectif et qualitatif de l'acteur sportif et, de l'autre, l'appréciation visuelle apparemment quantitative du système qui l'apprécie » (Carrier, 2002). 


\section{3. Une option didactique alternative}

L'ingénierie didactique que nous proposons s'appuie sur la mise à l'épreuve d'une option didactique alternative : elle vise l'élaboration par l'élève d'un savoir combattre, intégrant les savoirs chuter et projeter, lui permettant de prendre, de conserver ou de reprendre l'initiative dans chacun de ses combats réalisés avec des adversaires issus de sa classe. La perception, par l'élève, d'un "pouvoir combattre ", soit la compétence à s'engager avec/contre l'autre dans un combat de plus en plus exigeant, peut devenir l'enjeu d'un cycle de sport de combat. S'il veut éprouver sa "volonté de vaincre " (MEN, 1997), il lui faut prendre l'initiative.

C'est la construction de cette compétence qui constitue la trame de notre option. Une évaluation diagnostique en situation de combat simplifié (le sumo) permet d'identifier le savoir combattre " déjà là » des élèves en début de cycle. Les principes de (re)prise et de conservation d'initiative sont alors identifiés, expérimentés, puis complexifiés " avec/contre " (Bouet, 1969) ses partenaires. Dans le processus de construction de compétences, la situation d'interaction assure diverses fonctions :

l'interaction est fondatrice de la logique de contre-communication de l'activité dans la mesure où l'autre donne du sens aux conduites motrices ;

l'interaction facilite la co-construction d'habiletés motrices par le jeu de la coévaluation ou de la confrontation de solutions contradictoires.

La coordination de ses actions avec celles d'autrui dans une perspective constructive autoriserait de manière conjuguée des progrès moteurs et un engagement de plus en plus complexe du sujet en situation de combat.

Ici, nous faisons référence à la psychologie sociale du développement cognitif (Vygotsky, 1934 ) et plus particulièrement au concept de causalité "spiralaire ». Les progrès concernant les acquisitions dites "techniques " (référées à des critères de réussites et non à des modèles de formes de corps) sont liés au développement d'interactions sociales plus élaborées. C'est parce qu'il y a une co-élaboration du savoir que l'engagement, avec l'autre $\left({ }^{*}\right)$, dans des contextes d'opposition plus exigeants est possible, et inversement. La construction et l'actualisation d'un "savoir combattre " et celle d'un "pouvoir combattre " sont donc imbriquées. Ils singularisent la situation de combat pour le combattant. Ils ne sont pas sous la seule dépendance du contexte (et de sa perception par le combattant), mais révèlent aussi et avant tout la « logique d'action » (Bertaux, 1997) du sujet face à une situation qui peut s'avérer conflictuelle. 


\section{Méthodologie}

Nous choisissons d'adopter une méthode d'ingénierie didactique développée par Artigue dans le domaine des mathématiques (Artigue, 1988) et utilisée, après quelques modifications, dans le domaine de l'EPS (Amade-Escot, Marsenach, 1995 ; Loquet, 1996 ; Terrisse, Sauvegrain, 1997 ; Sauvegrain, 2000). Nous tentons de décrire, de comprendre, voire d'expliquer ce qui se passe lors d'un cycle d'enseignement du judo effectué par un enseignant d'EPS dans une classe de lycée professionnel.

Pour rendre compte du processus d'enseignement/apprentissage du savoir, nous suivons pas à pas les quatre étapes des ingénieries didactiques :

- les analyses préalables nous permettent, en prenant appui sur les connaissances actuelles concernant le judo et son enseignement, de justifier le choix de la notion d'initiative comme savoir à enseigner ;

- l'analyse a priori consiste ensuite à définir les contenus. Ces derniers sont orientés par les conclusions des analyses préalables et négociés avec l'enseignant responsable de l'enseignement ;

- La mise à l'épreuve permet de décrire ce qui se passe réellement en classe. C'est le moment de prélever des données concernant les réalisations d'élèves ;

- L'analyse a posteriori permet l'identification du savoir mis en jeu par les élèves lors de l'épreuve et la confrontation aux prévisions définies par l'analyse a priori. Nous recherchons alors « une causalité locale contextuelle " (Van der Maren, 1996).

Nous utilisons ce cadre de recherche dans une perspective clinique, c'est à dire s'intéressant au " cas par cas " (Terrisse, 1999). La nature de l'objet d'enseignement et l'intention de recherche ne peut se satisfaire d'une comparaison entre le savoir enseigné et le savoir appris. II nous faut adopter une posture de recherche différente et utiliser des outils qui nous permettent, "au cas par cas", de nous "rapprocher au plus près du monde intérieur des représentations et de l'intentionnalité des acteurs" (Van der Maren, 1996). L'analyse de la conduite de l'élève suppose de « la décrire en détail et, par un jeu subtil de regroupements et de recoupements dont les règles ne sont pas toujours fixées de façon explicite, faire apparaître une signification qui n'était directement lisible ni pour l'observateur profane ni, a fortiori, pour le sujet conscient lui-même " (Gréco, 1995). Nous avons choisi de rendre compte de l'activité du sujet en situation, mais aussi d'entendre ce que le sujet peut en dire.

Quatre types d'outils sont utilisés :

- Des questionnaires préliminaires sont proposés aux élèves afin de nous permettre d'identifier leurs représentations. 
- Les enregistrements vidéo permettent de fixer les réalisations de chacun des élèves choisis pour l'expérimentation. Deux temps sont retenus : l'évaluation diagnostique, soit le premier combat de sumo, et les combats de fin de cycle, supports de l'épreuve. « Une description précise s'avère indispensable si l'on veut comprendre un phénomène », nous dit Piéron (1993). Une grille nous permet de collecter des données caractérisant les comportements des deux combattants tout au long du combat. L'analyse porte sur chacune des périodes (pouvant être assimilée à des rounds en Boxe) rythmant le combat ; celles-ci sont délimités par le « hajimé » (commencez !) et le « maté » (arrêtez !) de l'arbitre et/ou par l'atteinte d'un résultat significatif.

Nous avons, ensuite, recours à de deux types d'entretiens :

- Les interviews « à chaud » réalisées à la fin du combat respectent un script défini. Pour tenter de réduire la recherche de conformité avec ce qui était attendu par l'enseignant et/ou le chercheur, ces interviews sont dirigés par un élève dispensé.

- Des entretiens semi-directifs de recherche (Blanchet, 1985) sont réalisés avec chaque élève une semaine après la fin du cycle. Ils sont étayés, dans la deuxième partie de l'entretien, d'un rappel stimulé (De Landsheere, 1979 ; Piéron, 1993) par la retransmission vidéo d'une partie de leur pratique de combattant. Ils sont réalisés avec l'enseignant avant le cycle, pendant le cycle et à l'issue du cycle. La première étape vise la proposition, puis la négociation du projet de cycle, compatible avec l'objet de la recherche et la dynamique de l'enseignant. Le deuxième temps a une fonction de régulation de ce projet en fonction des difficultés rencontrées. Enfin, l'entretien post-cycle a pour vocation de recueillir des éléments d'évaluation a posteriori, concernant les caractéristiques des élèves et la conduite de son cycle.

\section{Résultats et interprétation}

6. 1. L'initiative, la technique et la performance de chacun des élèves Les repères quantitatifs suivants n'ont pas pour vocation de comparer les productions de ces élèves avec d'autres ayant vécu une autre démarche d'apprentissage. L'effectif (quatre élèves) et le nombre de combats sur lesquels reposent ces observations ne peuvent, en outre, leur donner une pertinence statistique significative. Ces réalisations d'élèves produites lors de combats d'une durée de 2 minutes permettent, en premier lieu, d'apprécier la faisabilité et la pertinence de l'option didactique mise en scène par cet enseignant dans le contexte d'enseignement spécifique de cette classe.

Nous avons d'abord constaté que tous les élèves de la classe se sont engagés dans des combats, durant lesquels les projections étaient recherchées dans la limite de certaines 
contraintes préalablement définies et justifiées pour un premier niveau de pratique (Galan, 2000). Cet aspect est essentiel puisque seuls les élèves qui répondaient aux exigences (contrôlées par l'enseignant) des savoirs « chuter et projeter », et s'estimaient capables de combattre, pouvaient participer à cette épreuve.

La représentation graphique suivante nous permet de positionner les quatre élèves sur un plan factoriel qui consiste à croiser certains éléments « techniques » avec deux types de données prélevées dans les tableaux intitulés « l'initiative et sa gestion ».

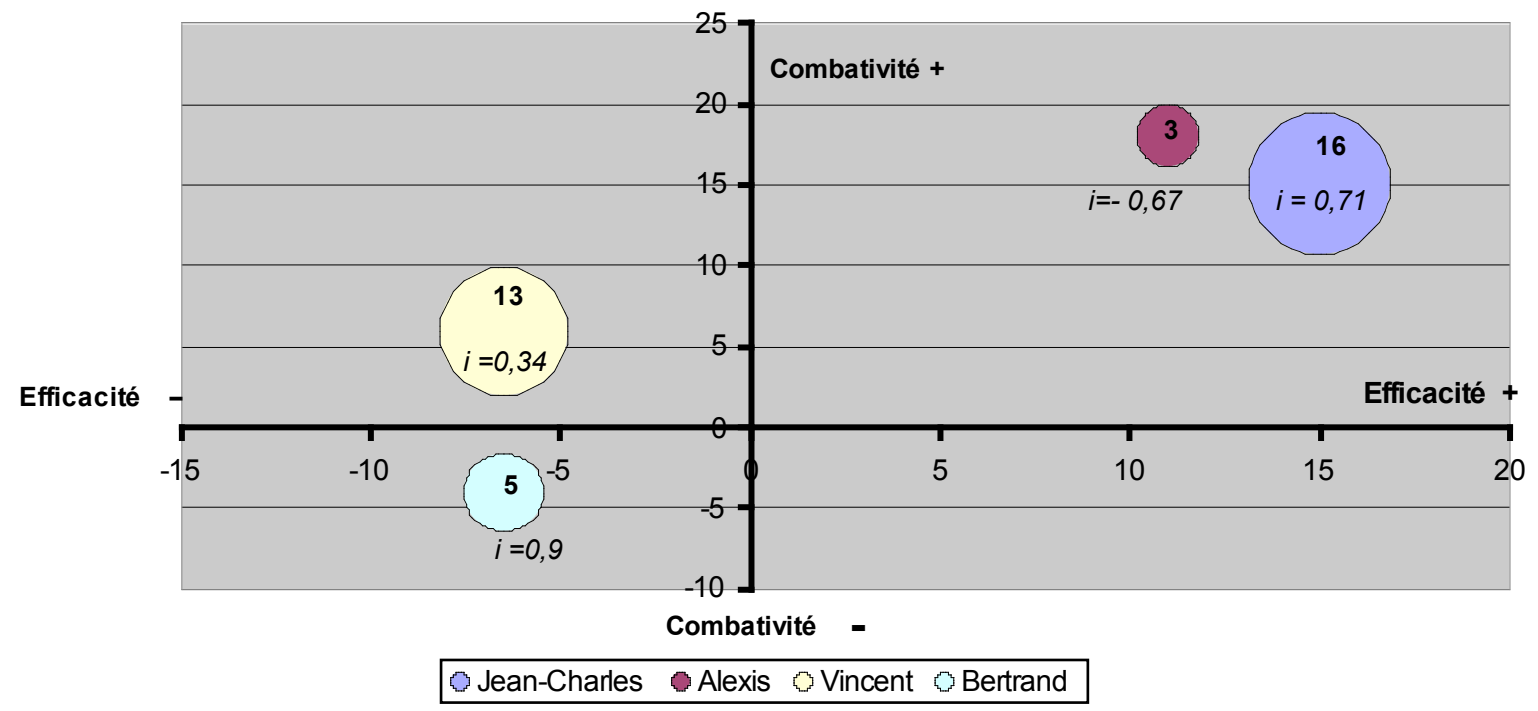

Figure1. La prise d'initiative, la technique et la performance

Le premier axe intitulé " efficacité » est une échelle sur laquelle sont inscrits les scores obtenus à l'issue des actions (projections, sorties) qu'ils ont entreprises. Le deuxième axe intitulé " combativité » indique le nombre de fois où ces élèves prennent l'initiative dans les combats. La dimension des «bulles » dépend du nombre de projections (noté en gras) tentées par les élèves sur l'ensemble de leurs combats. Un indice d'efficacité technique (noté en italique) leur est affecté ; il correspond au rapport du score obtenu grâce aux techniques par le nombre total de prises d'initiative.

Les données sélectionnées nous permettent de repérer comment ces élèves ont investi certains des contenus d'enseignement proposés pour faire face à l'épreuve. Elles nous permettent d'introduire un aperçu de la variété des parcours de chacun des élèves. D'emblée, nous remarquons que le nombre de prises d'initiative est associé à la dimension générale « efficacité » et n'est pas antinomique avec la dimension technique de l'activité. Notre étude nous permet de montrer que l'utilisation différenciée des principes de prises d'initiative n'est opposée ni au registre de l'efficacité, ni à celui de "l'expertise » 
technique. II nous faut maintenant envisager une « interprétation plausible » (Charlot, 1997) de ces cheminements singuliers.

Jean-Charles et Vincent sont ceux qui utilisent majoritairement ou exclusivement les projections pour tenter de marquer. Ils semblent s'inscrire dans la dynamique d'un combat de travail (le randori) sans exclure la recherche d'efficacité. Leurs tentatives bénéficient d'un indice d'efficacité technique positif. Jean-Charles est, dans ce secteur, deux fois plus efficace que Vincent. II est aussi celui qui témoigne de l'efficacité générale la plus importante. II associe les actions de sorties de tapis à ses tentatives de projection pour être efficace. Vincent n'utilise que les projections pour (re)prendre l'initiative et tenter de marquer.

Alexis et Bertrand utilisent peu de projections pour faire face aux exigences des combats. Le combat semble être, pour eux, une opération de mesure interpersonnelle (le shiaï) où la victoire semble l'enjeu unique. Ils se différencient par l'indice d'efficacité technique et le score qui représente l'efficacité totale pendant les combats. Bertrand a un indice d'efficacité technique important, mais un score d'efficacité faible. En fait, il semble adopter une logique binaire. Dans le cas, où il se représente en situation difficile, il ne produit rien ou peu de choses. Mais si le contexte lui est favorable, il peut alors investir le combat et faire montre d'un savoir appris en cohérence avec les contenus enseignés. A l'opposé, Alexis a un taux de réussite technique négatif, mais un score d'efficacité élevé. L'épreuve semble uniquement comprise dans sa dynamique compétitive; il faut vaincre! Les tentatives de projections sont reléguées à une part accessoire dans son savoir combattre, ce qui donne lieu à une recherche quasi-exclusive de sorties de tapis.

Pour synthétiser cette première partie de l'analyse des résultats, nous choisissons d'utiliser un tableau représentant « l'éventail des réponses » (Terrisse, 2000) associant les prises d'initiative aux réalisations techniques des élèves.

Tableau I. L'éventail des réponses des élèves confrontés à l'épreuve

\begin{tabular}{|l|l|l|}
\hline & $\begin{array}{l}\text { ADEQUATION à } \\
\text { l'épreuve }\end{array}$ & $\begin{array}{l}\text { INADEQUATION } \\
\text { à l'épreuve }\end{array}$ \\
\hline $\begin{array}{l}\text { En conformité, avec le savoir attendu par } \\
\text { l'enseignant }\end{array}$ & $\begin{array}{l}\text { Jean-Charles } \\
\text { l'initiative intégrative de } \\
\text { savoirs multiples }\end{array}$ & $\begin{array}{l}\text { Vincent } \\
\text { l'initiative idéalisée, } \\
\text { techno-centrée }\end{array}$ \\
\hline $\begin{array}{l}\text { En rupture, avec le savoir attendu par } \\
\text { l'enseignant }\end{array}$ & $\begin{array}{l}\text { Alexis } \\
\text { l'initiative non partagée et } \\
\text { ego déterminée }\end{array}$ & $\begin{array}{l}\text { l'initiative sous } \\
\text { conditions affectives }\end{array}$ \\
\hline
\end{tabular}


Les réalisations de l'élève sont appréciées en conformité ou en rupture par rapport au savoir attendu par l'enseignant. Ce savoir attendu est ici un score positif concernant les prises d'initiative associées à un nombre de tentatives de projections importantes.

Les réponses de l'élève sont adéquates à l'épreuve si le score d'efficacité de l'élève est positif.

Ces données nous permettent de cibler de manière macroscopique et externe le fonctionnement du sujet pendant le combat. Toutefois, la définition de l'objet d'enseignement en terme d'initiative pose avec force la place du sujet dans ses réalisations. Ainsi, il devient hypothétique de rechercher une conformité linéaire entre ce qui est attendu du point de vue de l'enseignant et ce que l'élève met en oeuvre en situation de combat. En effet, « le sujet n'est pas une distance vis-à-vis du social, il est un être singulier qui s'approprie le social sous une forme spécifique transmuée en représentations, en comportements, en aspirations, en pratiques, etc. En ce sens, le sujet a une réalité sociale, qui peut être étudiée, analysée, autrement qu'en terme de différence ou de distance » (Charlot, 1997).

L'étude de cas de ces élèves nous donne l'occasion d'explorer et de rendre compte de la dynamique de chacun des sujets au-delà des seules notions d'écart ou de positionnement.

5. 2. Les études de cas, l'articulation du dire et du faire

Puisque seul le sujet peut prendre l'initiative et répondre de ses choix, nous avons choisi de nous intéresser à ce que fait et dit l'élève. Cette position épistémologique constitue l'originalité de la référence à ce travail clinique, en ce sens qu'au delà de la compétence de l'enseignant à reconstruire le sens de l'activité et des transformation visées par les situations d'apprentissage qu'il met en place, seul l'élève, s'il l'accepte, est en mesure de renseigner le chercheur sur les décisions qu'il a prises en combat en terme d'initiatives. Certes, comme tout sujet, il n'est pas toujours en mesure de les formaliser. C'est la raison pour laquelle, les conditions méthodologiques de recueil et de traitement des données ont été construites dans ce sens, celui de rendre compte de ses choix et justifications. Le savoir combattre mis en œuvre lors de l'épreuve dépend du sens que l'élève accorde aux différents savoirs, à la situation de combat, aux autres et à soi-même. "Le système de finalités, propre à chaque acteur ", dans le cadre d'une situation de combat vécue singulièrement, est ici " considéré comme le déterminant fondamental de son action » (Raisky, 1999).

En effet, Charlot (op.cit) rappelle que l'élève fait bien autre chose qu'intérioriser les savoirs enseignés et autres éléments issus de la situation didactique, il se les approprie. La mobilisation des savoirs (" déjà-là », appris, de l'épreuve) au moment de l'épreuve est, 
avant tout, générée par l'activité d'un sujet confronté à la nécessité d'apprendre et de faire montre de ses apprentissages, dans un contexte particulier, le combat de judo qui le met en relation avec un partenaire lui aussi singulier.

Notre recherche s'appuie, certes, sur des repères (savoir faire, "savoir y faire » (Terrisse A, 2002 , mais suppose de se détacher du produit (les comportements) pour tenter de comprendre la logique d'action de l'élève. Ces repères constituent quelques attentes, «quelques limites quant (...) aux prestations fournies » mais « ne délivrent aucune prescription quant au travail que celui » qui combat « doit accomplir pour réussir, c'est à dire pour atteindre les objectifs qu'il s'est fixé »(Raisky, op.cit). L'étude de cas semble fournir un cadre d'analyse fécond permettant de confronter le dire et le faire des sujets, pour accéder à un niveau de compréhension différent de la dynamique de l'élève. Cette posture de recherche nous permet de nous détacher des seules informations issues de la situation de combat, en se centrant sur ce qu'en fait le sujet qui est le mieux placé pour « dire quelque chose sur la part qu'il prend dans ce qui lui arrive » (Terrisse, 2000).

Nous choisissons ci-dessous d'évoquer les grandes lignes de deux études de cas afin de préciser l'intérêt de « l'après coup » pour le chercheur et pour le sujet.

Alexis apparaît en rupture avec le savoir attendu par l'enseignant et réussit toutefois au moment du combat.

Jean-Charles réussit lui aussi lors de l'épreuve, et mobilise un savoir en conformité avec le savoir attendu par l'enseignant.

5. 2. 1. L'après coup, ou l'accès à une signification différente pour l'observateur Premier cas : Alexis ( je faisais comme il me semblait bon »)

L'enseignant le situe " en frange de l'institution scolaire, de ses contraintes et de ses règles ». Dans ses combats, il adopte (quel que soit l'adversaire) une logique combative interdisant les prises d'initiative à son adversaire. Notre première évaluation indique qu'Alexis est centré sur lui-même et sur le résultat. II s'appuie sur un « déjà là » suffisant pour faire face efficacement à l'épreuve et toujours à la limite de ce qui est permis (« il n'a rien tenté ", "il triche, il a toujours les bras tendus" (commentaire de l'un de ses adversaires). II gagne par la seule mise en jeu de sa force (principe d'initiative P1), et de manière quasi systématique par le biais d'une poussée de l'adversaire hors des limites du tatami ( je faisais comme il me semblait bon »). En cela, il ne semble pas faire appel au savoir enseigné, ce qui produit un effet de rupture avec le travail attendu par l'enseignant. Pourtant, l'entretien de fin de cycle permet d'inscrire ces comportements dans un registre explicatif différent. Une part de son histoire (qu'il nous livre) expliquerait ses attitudes et comportements de mise à distance des autres ( " eh bien, j'essaie de le pousser loin de 
moi, de tendre mes bras, comme ça il peut moins m'attraper et puis j'essaie de le pousser, pas de le faire tomber parce que si je lui tombe dessus aussi... »), moins dans le but de s'inscrire en marge de ce qui est attendu que de protéger l'autre et lui-même ( "j'ai pas envie de me faire mal, ni de faire mal »)... II a en effet vécu une expérience douloureuse de la pratique du judo en club (une saison à l'âge de dix ans) : "j'ai trouvé ça dur, ils y allaient vraiment comme des ânes dans le club où j'étais, donc ça m'a fait peur, je n'avais pas envie de recommencer ça ".

5. 2. 2. L'après coup, l'occasion de remaniement pour le sujet Deuxième cas : Jean Charles (« je fais ce qu'on me demande de faire ») La posture clinique, les outils et les temps requis de cette investigation constituent, pour Jean-Charles, les conditions de remaniement de ses représentations de lui-même et de ses savoirs.

Jean Charles est présenté par l'enseignant comme " un élève appliqué et en réussite » en éducation physique et sportive. La première partie de l'entretien lui permet d'exprimer cet assujettissement à l'institution scolaire, via la référence au savoir enseigné ( « ...j'essaie de montrer ce que j'ai appris »). Effectivement, nous percevons dans ce que produit JeanCharles certaines régularités concernant le respect des consignes de sécurité inhérentes aux chutes et aux projections, la propension à tenter davantage de projections que de sorties, ainsi que l'orientation de ces tentatives de projections vers plusieurs secteurs de projections. Mais il est aussi capable de différencier ses actions, selon les adversaires ou les contextes de combat, afin de prendre, reprendre ou conserver l'initiative.

L'observation de la vidéo lui donne l'occasion d'accéder à la richesse de son savoir combattre, de faire des liens entre sa perception de l'adversité, sa re-connaissance du problème posé (" il me chargeait, il était fort... ») et la solution qui semble adaptée (" alors je me suis servi de sa force ») puis d'effectuer une synthèse de l'activité qui pourrait constituer un modèle de définition de ce que les judokas doivent faire en randori : ( « il (le partenaire) sert à se battre, à s'exprimer, à chacun de nous deux, montrer ce qu'on sait faire chacun de nous deux »).

\section{Conclusions de la recherche}

L'épreuve est donc bien à concevoir dans une perspective individualisée, elle fait sens de manière spécifique pour chacun des élèves, comme le montrent les entretiens au delà des analyses vidéo. Leurs réponses dépendent de registres explicatifs multiples difficilement prévisibles. Si les repères sont nécessaires pour celui qui veut rendre compte et/ou faire évoluer les conduites de sujets apprenants, il semble nécessaire d'introduire des espaces 
et moyens permettant à l'élève d'investir le combat de judo sur « un mode d'être concordant à son mode actif » Birouste (1991). Le sujet s'approprie alors par sa pratique ses « codes et habitus sociaux" (op.cit) tandis que ceux-là contribuent à le transformer.

La définition du savoir en terme d'initiative semble favoriser le processus d'interstructuration du sujet, de l'épreuve et des savoirs attendus par l'enseignant. Le sujet investit d'autant plus facilement l'épreuve et les savoirs qui lui sont associés qu'il les perçoit compatibles avec son économie singulière.

L'adoption d'une posture épistémologique et méthodologique permettant de mettre en avant « ce que les gens font, réussissent, ont et sont et non pas à ce qu'ils ratent et à ce qui leur manque » (Charlot, 1997) semble nécessaire au chercheur et/ou à l'enseignant s'ils désirent rendre compte de (voire favoriser) la dynamique du sujet confronté à la nécessité d'apprendre.

Cette recherche a permis de montrer la « faisabilité » d'une telle option didactique et de ses effets en terme d'initiatives d'élèves. Ainsi, il ne s'agissait pas de s'engager dans une démarche d'établissement de preuve, mais dans la recherche d'une «causalité locale contextuelle »(Van Der Maren, 1996).

\section{Perspectives didactiques}

Notre recherche n'a donc pas vocation prescriptive. Toutefois, nous proposons quelques orientations susceptibles de favoriser les progrès chez les élèves. Une nécessité apparaît pour l'enseignant qui veut conduire chacun de ses élèves à infléchir sa logique d'action vers un « toujours mieux » : celle de les confronter, en se reconnaissant " responsable » (Brousseau, 1986), à l'usure du savoir antérieur, afin de le dépasser. Plusieurs facteurs sont alors à prendre en compte :

- Le choix des partenaires influe sur les modalités de prises d'initiative ; ils constituent une ressource permettant la mise à l'épreuve du savoir combattre de l'élève (cas de Bertrand lorsqu'il change de partenaire).

- La définition de l'épreuve est essentielle pour orienter l'engagement des combattants. Lors de ce cycle, les contraintes de sécurité lors des projections et le système de marque de points semblent contribuer au développement par l'élève de compétences et de savoirs spécifiques témoignant de l'appropriation d'une « culture judo ».

- L'adoption d'un référentiel d'évaluation « prospectif » intégrant à la fois le système de comptabilité validant l'efficacité, et des critères de réussite qualifiant leur tentatives de (re)prises d'initiative pourrait venir soutenir l'infléchissement de leurs attitudes spontanées 
vers le savoir attendu de l'enseignant, sans pour autant empêcher l'invention (exemple, pour Alexis, adopter des registres de prises d'initiative plus complexes).

- La connaissance des résultats semble un facteur de renouvellement de l'évaluation de soi effectuée par l'élève. L'analyse en terme d'initiative de tous les comportements identifiés lors de l'épreuve pourrait ensuite lui permettre de les complexifier. En appréciant ce qu'il réalise à la première personne, « Je », (c f : étude de cas de Jean Charles) l'élève se met au centre de la situation ; il ne se réduit pas aux seuls comportements qu'il met en oeuvre: Est-ce que l'action que j'entreprends me permet de marquer, de prendre, reprendre ou conserver l'initiative? Ce questionnement peut donner corps à la construction d'une démarche réflexive permettant de « mesurer et apprécier les effets de l'activité » (B.O.E.N., 2000). Ainsi, le thème d'initiative peut induire l'élaboration d'un savoir combattre singulier, original, pouvant être compatible avec l'économie du sujet et les attentes de l'enseignant tout en étant pertinent en regard des exigences du combat avec/contre un autre.

\section{Bibliographie}

Alberti, C., Sauret, M.J. (1997). La psychanalyse, les essentiels, Milan.

Amade-Escot, C., Marsenach, J. (1995). "Recherche d'ingénierie didactique », in Didactique de l'éducation physique et sportive, La pensée sauvage, Grenoble.Artigue, M. (1988). "Ingénierie didactique », Recherche en didactique des mathématiques, $n^{\circ} 9,283-307$.

Avanzini, G. (1986). A propos de la didactique : il n'y a pas consensus. Le binet Simon, 606, pp.3-12.

Badreau, J. (1994). Transposition didactique: pour une analyse fonctionnelle des savoirs sportifs. In dossier EPS n¹9, techniques sportives et EP, Ed. Revue EPS, 76-79. Barbot, A. (1996). Approche technologique des phénomènes d'enseignement du Judo en EPS, thèse non publiée, Université Paris XI Orsay.

Bertaux, D. (1997). Les récits de vie, Paris, Nathan.

Birouste, J. (1991). Economie pulsionnelle du goût des sportifs. in J. Bilard et M. Durand (Eds), Sport et psychologie, Dossiers EPS $n^{\circ} 10$, actes du congrès international de psychologie du sport, octobre 1990, Montpellier : Ed. Revue EPS,367-372.

Blanchet, A. (1985). L'entretien dans les sciences sociales, Paris, Bordas.

Bouet, M. (1969). Les motivations des sportifs. Paris :ed. Universitaires, 145-158. 
Brousse, M. (1994). La technique ou l'ordre du combat. In techniques sportives et éducation physique. Dossier EPS n¹9.80-89.

Brousseau, G. (1986). Théorisation des phénomènes d'enseignement des mathématiques, thèse d'état non publiée, Université de Bordeaux I.

Bui-Xuan, G. (1993). Bande-Zaï : ou la santé par le jiu-jitsu. Revue quel corps $n^{\circ} 45$ et 46 , 75-81.

Calmet, M. (2000) Judo : la notion plurielle d'équilibre. In recherches en sports de combat et en arts martiaux, état des lieux. Paris, ed. Revue EPS, 275-282.

Carrier, C. (2002). Le sportif exemplaire: idole ou martyr ? in la fièvre du dopage. Les revers de la performance.

Charlot, B. (1997). Du rapport au savoir, Paris, Editions Economica.

De Landshere, G. (1979). Dictionnaire de l'évaluation et de la recherche en éducation, Paris, PUF.

Durand, M., Geoffroi, V., \& Jacquemond, L. (1993). Constance et stabilité des tâches, invariance des adaptations motrices et techniques sportives. In cognition et Performance. Paris, publications INSEP, 145-163.

Galan, M. (2000). Contribution à l'évaluation du judoka débutant. In recherches en sports de combat et en arts martiaux. Etat des lieux. Paris, ed. Revue EPS.

Giordan, A. (1999). EPS interroge un didacticien, revue EPS n²79, 13-18.

Gréco, P. (1995). L'optique clinique en psychologie. Encyclopédia Universalis.

Heuzer, F., Terrisse, A., \& Carnus, M.F. (2005) Ecart entre savoir enseigné et savoir appris en éducation physique et sportive:étude de cas en karaté. Ejrieps, Besançon.

Joshua, S. (1996). Le concept de transposition didactique n'est-il propre qu'aux mathématiques. In Au delà des didactiques, le didactique. Bruxelles, Ed. De Boeck.

Kano, J. (1956). Judo kodokan. Editions de l'éveil. (Ed . 2001).

Larousse. (2002). initiative.

Loizon, D. (2001). Analyse des pratiques d'enseignement du judo : identification du savoir transmis à travers les variables didactiques utilisées par les professeurs de judo. Mémoire non publié pour l'obtention du brevet d'Etat d'éducateur sportif $3^{\text {ème }}$ degré.

Loizon, D., Margnes E., \& Terrisse, A. (2004). Analyse des pratiques d'enseignement : les pratiques déclarées par les professeurs de judo. eJRIEPS, 5,, IUFM de Besançon. 
Loquet, M. (1996). Les contenus d'enseignement en GRS pour les élèves de sixième, étude des lancer-rattraper d'engin, une recherche d'ingénierie didactique, Thèse d'université, non publié, pour le doctorat de didactique des APS., Orsay.

M.E.N. (1997). Accompagnement des programmes de $6^{\text {ème }}, 5^{\text {ème }}$ et $4^{\text {ème }}$.

M.E.N. (2001). Programme d'EPS du cycle terminal des séries générales et technologiques.

Margnes, E. (2002). L'intention didactique dans l'enseignement du judo, des choix culturels, d'ordre éthique et technique. Thèse de doctorat, non publiée, Université de Toulouse III.

Marsenach, J. (1991). Education physique et sportive. Quel enseignement ?.Paris, INRP. Martinand, J.-L. (1989). "Pratiques de référence, transposition didactique et savoirs professionnels en sciences et techniques », in les sciences de l'éducation pour l'ère nouvelle, Caen.

Parlebas, P. (1981). Contribution a un lexique commente en science de l'action motrice, Paris, INSEP.

Parlebas, P. (1993). Une tribu au XXème siècle, revue EPS, 243.

Piéron, M. (1993). Analyser l'enseignement pour mieux enseigner, dossier EPS $n^{\circ} 16$, Paris, ed. revue EPS.

Raiski, C. (1999). Complexité et didactique. Education permanente. $\mathrm{n}^{\circ} 139$.

Rochex, J.-Y. (1995). Le sens de l'expérience scolaire, Paris, PUF.

Rougé, J.L.(1997). in Peressini, D., Gainier, G. Vidéo : histoire et légende du judo, saint louis production/ffj/France télévision distribution

Sauvegrain, J.-P. (2000). "Apprendre la défense plutôt que l'attaque ", in Terrisse A., (sous la direction de), recherches en sports de combat et en arts martiaux, Paris, ed. Revue EPS. Sauvegrain, J.-P., (2001). Analyse didactique de la décision de l'élève, étude de cas sur l'utilisation du savoir dans un cycle de lutte en EPS, thèse de doctorat, non publiée, université de Toulouse III.

Terrisse, A. (1996). « Analyse de la transposition didactique en judo : évolution du savoir combattre dans l'enseignement du judo a l'école a travers la revue EPS de1950 à $1993 »$, revue française de pédagogie, $n^{\circ} 116,23-38$.

Terrisse, A. (2000). "Epistémologie de la recherche clinique en sports de combat ", in Terrisse A., (sous la direction de), recherches en sports de combat et en arts martiaux, Paris, ed. Revue EPS. 
Terrisse, A. (2002). "L'extraction du savoir des entraîneurs: le point de vue de la psychanalyse », in Loquet, Léziart, Cultures sportives et artistiques : formalisation des savoirs professionnels, ARIS, Rennes.

Terrisse, A., Sauvegrain, J.-P. (1997). «Identification des difficultés d'appropriation du " savoir lutter " par une méthodologie d'ingénierie didactique ", Revue Science et Motricité, $n^{\circ}$ 32-33, 74-87.

Van Der Maren, J.-M. (1996). Méthodes de recherche pour l'Education, Bruxelles, ed. De Boeck.

Verret, M. (1975). Le temps des études. Thèse d'Etat Paris V, Paris.

Vigne, P. (1999). Activité sportive, conflit et personnalisation à l'adolescence. Thèse de Doctorat en Psychologie, non publiée, Université Toulouse le Mirail.

Vigotsky, L.S. (1934). Pensée et Langage. Paris, Flammarion. 